\title{
Short-range effects on nuclear pairing
}

\author{
W. Zuo ${ }^{1}$, U. Lombardo ${ }^{2,3}$, H.-J. Schulze ${ }^{4}$, and C. W. Shen ${ }^{3}$ \\ 1 Institute of Modern Physics, Lanzhou, China \\ 2 Dipartimento di Fisica, Università di Catania, 57 Corso Italia, I-95129 Catania, Italy \\ ${ }^{3}$ Laboratori Nazionali del Sud, Via Santa Sofia 44, I-95123 Catania, Italy \\ ${ }^{4}$ INFN, Sezione di Catania, 57 Corso Italia, I-95129 Catania, Italy
}

\begin{abstract}
We determine the influence of the three-body force and the medium modification of meson masses on pairing in nuclear and neutron matter. A reduction of the pairing gap is found and increasing with density.
\end{abstract}

PACS: 21.65.+f, 24.10.Cn 
The pairing interaction in electron superconductors has a natural cutoff determined by the lattice spacing. On the contrary, in nuclear matter there is no such sharp cutoff. The realistic bare nucleon-nucleon $(N N)$ interactions have a smooth behavior which is determined by the fit of the experimental phase shifts of high-energy nucleon-nucleon scattering. However, medium effects strongly modify the $N N$ interaction in nuclear and neutron matter, including its short-range part [1]. We have still a poor knowledge of these modifications. All predictions on the pairing gap in nuclear matter suffer from this drawback, as the solution of the gap equation is very sensitive to the value of the tail of the interaction in momentum space [2], i.e., to the short-range behavior of the coordinate-space potential.

In this note we discuss the role played in neutron and nuclear matter superfluidity by some mechanisms which may affect the short-range behavior of the pairing force. The nucleon-nucleon correlations (ladder diagrams), which renormalize the short-range part of the interaction much the same as in the Brueckner $G$-matrix, fix already a kind of cutoff in the gap equation [2]. Other processes may have a strong influence, such as nucleonic excitations or $\bar{N} N$ exchange giving rise to three-body forces (TBF) [3]. TBF have been proven to play a crucial role for the saturation properties of nuclear matter due to their short-range repulsive nature [4]. In addition, a medium modification of the heavy meson masses, which has been addressed as manifestation of the chiral symmetry restoration in nuclear matter [5], can modify the range of the interaction. Experimental evidence for the latter is provided by dilepton production in high-energy heavy ion collisions [6].

The magnitude of the pairing gap in nuclear matter is determined by the competition between the repulsive short-range part and the attractive long-range part of the interaction. The medium modification of the long-range part was confidently obtained within the RPA approximation or induced interaction model, and it was discussed elsewhere $[1,7,8]$. The short-range part is partially incorporated by the gap equation itself with the bare interaction. A simple way [2] to illustrate this property is to split the gap equation into two coupled equations,

$$
\begin{aligned}
\Delta_{\boldsymbol{k}} & =-\sum_{k^{\prime} \leq k_{c}} \widetilde{V}\left(\boldsymbol{k}, \boldsymbol{k}^{\prime}\right) \frac{\Delta_{\boldsymbol{k}^{\prime}}}{2 E_{\boldsymbol{k}^{\prime}}}, \\
\widetilde{V}\left(\boldsymbol{k}, \boldsymbol{k}^{\prime}\right) & =V\left(\boldsymbol{k}, \boldsymbol{k}^{\prime}\right)-\sum_{k^{\prime \prime} \geq k_{c}} \frac{V\left(\boldsymbol{k}, \boldsymbol{k}^{\prime \prime}\right) \widetilde{V}\left(\boldsymbol{k}^{\prime \prime}, \boldsymbol{k}^{\prime}\right)}{2 E_{\boldsymbol{k}^{\prime \prime}}},
\end{aligned}
$$

where $E_{\boldsymbol{k}}=\sqrt{\left(e_{\boldsymbol{k}}-\mu\right)^{2}+\Delta_{k}^{2}}$. This shows that the effective interaction $\widetilde{V}$, arising from the introduction of a cutoff $k_{c}$ in momentum space, sums up a series of ladder diagrams analogous to the Bethe-Goldstone equation. We want to stress that $\widetilde{V}$ and the cutoff are interrelated, so that one cannot use a phenomenological interaction in the gap equation and fix arbitrarily the cutoff. It is quite sensitive to the tail $\left(k>k_{c}\right)$ of the $N N$ interaction, which reflects the short-range part of the nuclear force.

A great deal of uncertainty is associated with the modification of the short-range part of the two-body interaction when going from the vacuum to the nuclear medium. It is well know that the empirical saturation properties of nuclear matter cannot be reproduced with the bare two-body force only, but that one has to include also three-body forces which take into account the medium modification of the in-vacuum $N N$ interaction [4]. The 
effect of the three-body forces around the saturation region is mainly to shift to lower density the balance between short-range repulsive and long-range attractive components of the interaction, which mostly dominate above the saturation density $\left(\rho_{0} \approx 0.15-0.17 \mathrm{fm}^{-3}\right)$. In the pairing problem we thus do not expect a remarkable effect in the peak domain of the energy gap $\left(\rho \approx 0.02 \mathrm{fm}^{-3}\right)$. In Fig. 1 we plot the effective two-body force in the ${ }^{1} S_{0}$ channel, obtained from a meson-exchange model of the three-body force [3]. The density dependence arises from the averaging procedure adopted to reduce the three-body force to an effective two-body force [4]. In neutron matter the strength of $V_{3}(r)$ is smaller than in nuclear matter due to the lack of the $T=0$ tensor force. In either case the strength of $V_{3}(r)$ is very small in comparison to the two-body force plotted also for comparison in Fig. 1. However, it is mainly effective on the short-range side $(r \lesssim 1 \mathrm{fm})$ and hence it affects $\tilde{V}$. The repulsion is increasing with rising density.

In order to numerically investigate the effect of the three-body force we have solved the gap equation, Eq. (1), adding to the bare two-body force (the Argonne $V_{18}$ potential [9]) the above three-body force. At the same time the single-particle spectrum $e_{\boldsymbol{k}}$ appearing in the gap equation is computed using the same three-body force. We have considered the two cases of ${ }^{1} S_{0}$ pairing between like nucleons embedded in neutron matter and nuclear matter. The results for the energy gap $\Delta_{F}=\Delta\left(k_{F}\right)$ are depicted in Fig. 2. The gap in nuclear matter is always smaller than in neutron matter, since the single-particle spectrum is different in the two cases [10]. As expected, the effect is a slight suppression of the gap, quasi negligible at low density and increasingly larger at higher density in both cases.

Since the masses of the exchanged mesons drive the range of the interaction, it is also of interest to discuss in this note to which extent the energy gap is influenced by the change of the properties of the heavy mesons which act at short distances. Unfortunately, at the present time this approach suffers a great deal of uncertainty, since there is no clear idea about the medium modification of the meson parameters. The latter is motivated as being due to the partial restoration of chiral symmetry, which causes hadron mass scaling [5] in the medium. It appears experimentally supported by the quenching of the $\rho$ meson mass in dilepton production in heavy ion collisions [6]. The theoretical treatment [11] can only be accomplished using an interaction explicitly dependent on the meson parameters. For this reason we use the Bonn-B potential [12] and only investigate the effect of the $\omega$ meson mass, whose strength is by far the largest one. The Bonn-B potential has also been used recently in the relativistic theory of pairing, where it predicts a gap comparable with the result of the nonrelativistic BCS theory [13].

We have done two sets of calculations: One is obtained by just changing the meson mass (and consistently the mass cutoff) in a range independent of the density; in the other one the change of the mass is connected to the density according to the Brown and Rho scaling model [5]:

$$
\frac{m_{\omega}^{*}}{m_{\omega}}=\frac{\Lambda_{\omega}^{*}}{\Lambda_{\omega}}=\frac{1}{1+\alpha\left(\rho / \rho_{0}\right)},
$$

where $\alpha$ is a constant and $\Lambda$ is the mass cutoff. This scaling of hadron masses has been proposed to partially restore the chiral symmetry of the QCD Lagrangian in nuclear matter. In Fig. 3 the results are plotted for the two sets of calculations. In order to demonstate the qualitative effect we solve the gap equation with a free single-particle spectrum. The 
Bonn-B potential has been used as pairing interaction with all meson parameters frozen except for the $\omega$ meson. On the left side of the figure the first set of results is plotted. Changing the mass of the $\omega$ meson independently of the neutron density amounts to the same change of the range of the interaction and therefore a deviation of the pairing gap from the original curve is found at any density. It is worth noticing that decreasing the $\omega$ meson mass the peak value of the energy gap shifts to slightly lower densities, because the average distance of the competition between long-range and short-range components of the interaction increases and hence the pairing peak shifts to a lower density. On the right side of the figure the deviation of the gap from the original value is increasing with density simply because the reduction of the $\omega$ mass also increases. The effects is stronger for higher scaling parameter. The shift of the peak value has the same explanation as earlier.

In summary, we have discussed the influence of the short-range part of the interaction on the ${ }^{1} S_{0}$ pairing gap in neutron matter. Mechanisms which come into play at short distance, such as particle-particle ladder correlations, three-body forces, and meson masses, have been discussed in the solution of the BCS gap equation. These effects appear to point altogether to a reduction of the gap which seems however small compared to that effected by polarization corrections to the interaction [1]. Unfortunately, at the present time no definite quantitative conclusions can be drawn due to too many theoretical incertainties.

\section{ACKNOWLEDGMENTS}

We are indebted to Prof. J. W. Clark for drawing our attention to the study of the three-body force in relation with pairing. One of us (W. Z.) would like to thank INFN-LNS and the Physics Department of the Catania University (Italy) for their hospitality during the preparation of the present work. This work has been supported in part by the Chinese Academy of Science within the one Hundred Person Project, Knowledge Innovation Project (No. KJCX2-SW-N02), and the Major State Basic Research Development Program of China under No. G2000077400. 


\section{REFERENCES}

[1] U. Lombardo and H.-J. Schulze, "Superfluidity in Neutron Star Matter" in Physics of Neutron Star Interiors, Lecture Notes in Physics vol. 578, pp. 30-54, eds. D. Blaschke, N. K. Glendenning, and A. Sedrakian (Springer, 2001).

[2] U. Lombardo, "Superfluidity in Nuclear Matter" in Nuclear Methods and the Nuclear Equation of State, Ed. M. Baldo, (World Scientific, Singapore, 1999), Chapt. 9, p. 458.

[3] P. Grangé, A. Lejeune, M. Martzolff, and J.-F. Mathiot, Phys. Rev. C40, 1040 (1989).

[4] A. Lejeune, U. Lombardo, and W. Zuo, Phys. Lett. B477, 45 (2000); W. Zuo, A. Lejeune, U. Lombardo, and J.-F. Mathiot, Nucl. Phys. A706, 418 (2002).

[5] G. E. Brown and M. Rho, Phys. Rev. Lett. 66, 2720 (1991).

[6] CERES Collaboration, G. Agakichiev et al., Phys. Rev. Lett. 75, 1272 (1995); Nucl. Phys. A661, 23c (1999).

[7] J. M. C. Chen, J. W. Clark, R. D. Davé, and V. V. Khodel, Nucl. Phys. A555, 59 (1993).

[8] H.-J. Schulze, J. Cugnon, A. Lejeune, M. Baldo, and U. Lombardo, Phys. Lett. B375, (1996) 1.

[9] R. B. Wiringa, V. G. J. Stoks, and R. Schiavilla, Phys. Rev. C51, 38 (1995).

[10] M. Baldo, J. Cugnon, A. Lejeune, and U. Lombardo, Nucl. Phys. A515, (1990) 409.

[11] A. B. Santra and U. Lombardo, Phys. Rev. C62, 18202 (2000).

[12] R. Machleidt, Adv. Nucl. Phys. 19, 189 (1989).

[13] M. Serra, A. Rummel, and P. Ring, Phys. Rev. C65, 014304 (2001). 


\section{FIGURES}

FIG. 1. Nucleon-nucleon interactions vs. distance. The dashed curve shows the Argonne $V_{18}$ two-body force. Solid curves with different symbols display the effective three-body force (scale enlarged by a factor 10) in neutron matter (left panel) and symmetric nuclear matter (right panel) at different neutron Fermi momenta, as indicated.

FIG. 2. ${ }^{1} S_{0}$ gap in neutron matter and in symmetric nuclear matter. Solid curves show the results obtained using $A V_{18}$ plus three-body force. Dashed curves are calculated adopting the pure $A V_{18}$ two-body force. The dotted curve indicates the result using a free single-particle spectrum and the bare $A V_{18}$ force. 
FIG. 3. ${ }^{1} S_{0}$ pairing gap in neutron matter calculated using the Bonn-B potential with scaled $\omega$ meson mass and free single-particle energy spectrum. Left panel: results with different $\omega$ meson mass ratios $M_{\omega}^{*} / M_{\omega}=\Lambda_{\omega}^{*} / \Lambda_{\omega}$, indicated near the corresponding curves. The solid curve corresponds to unit ratio. Right panel: results according to Brown-Rho scaling with different values of the scaling parameter $\alpha$. 

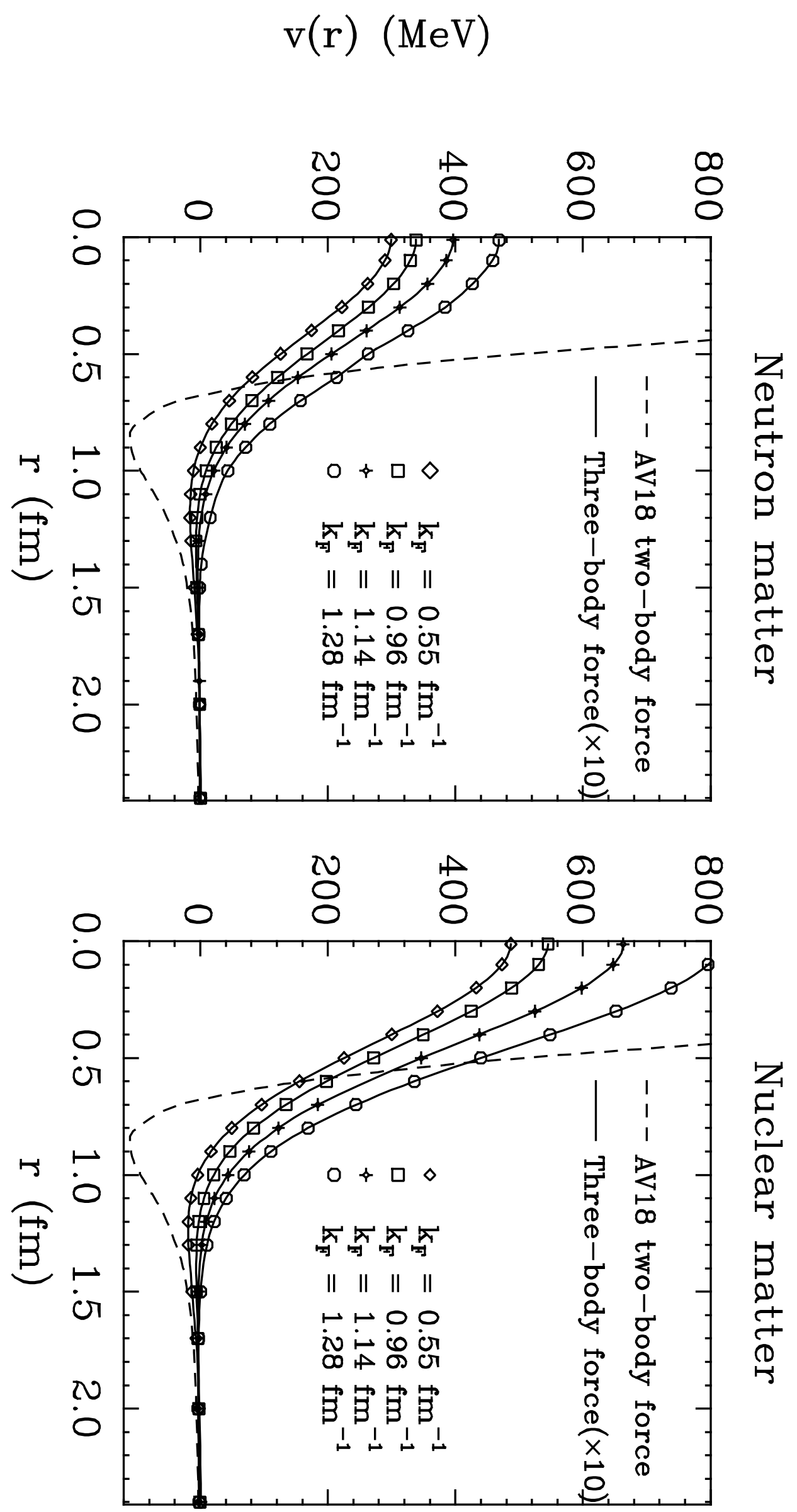


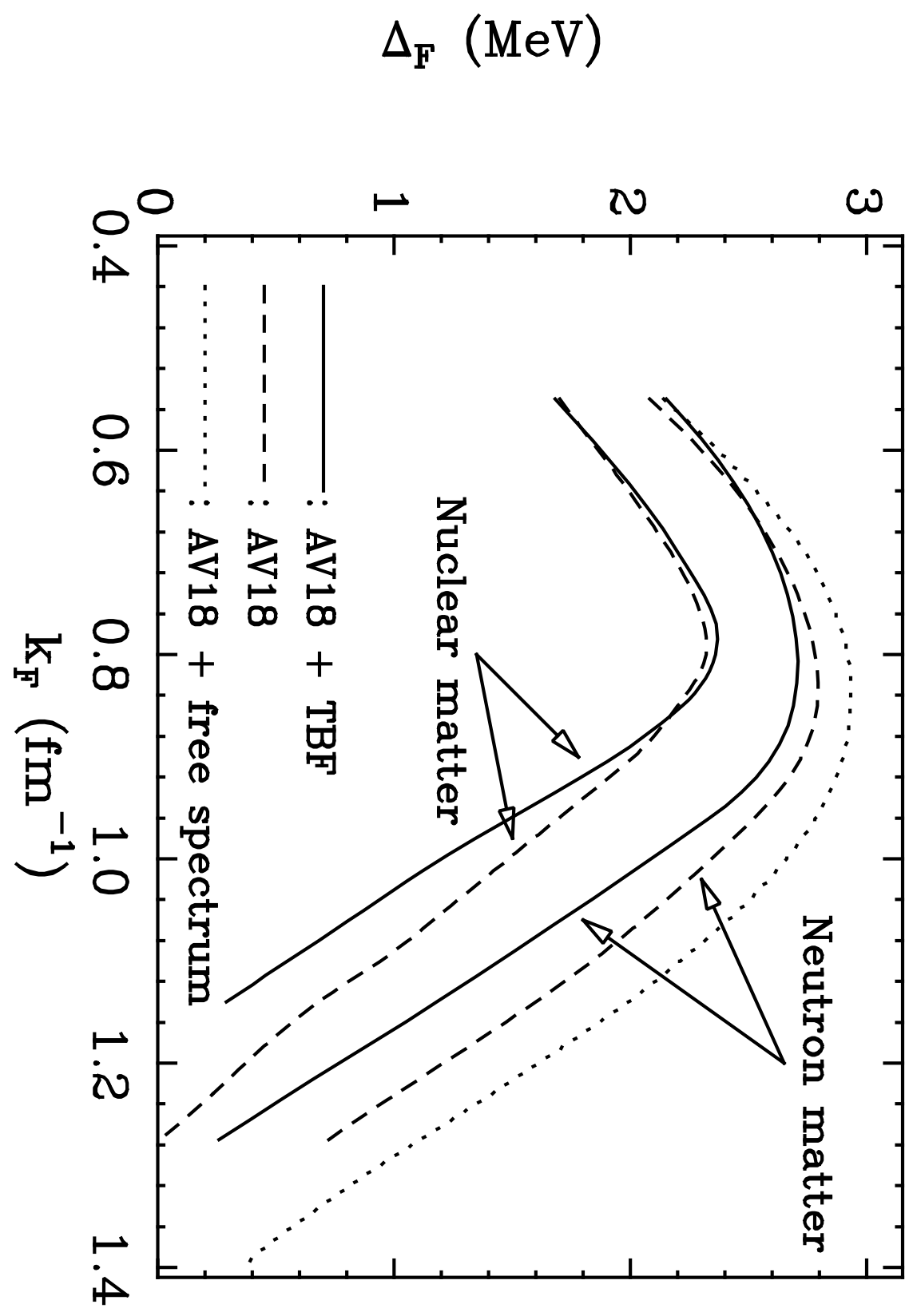


$\Delta_{\mathrm{F}}(\mathrm{MeV})$
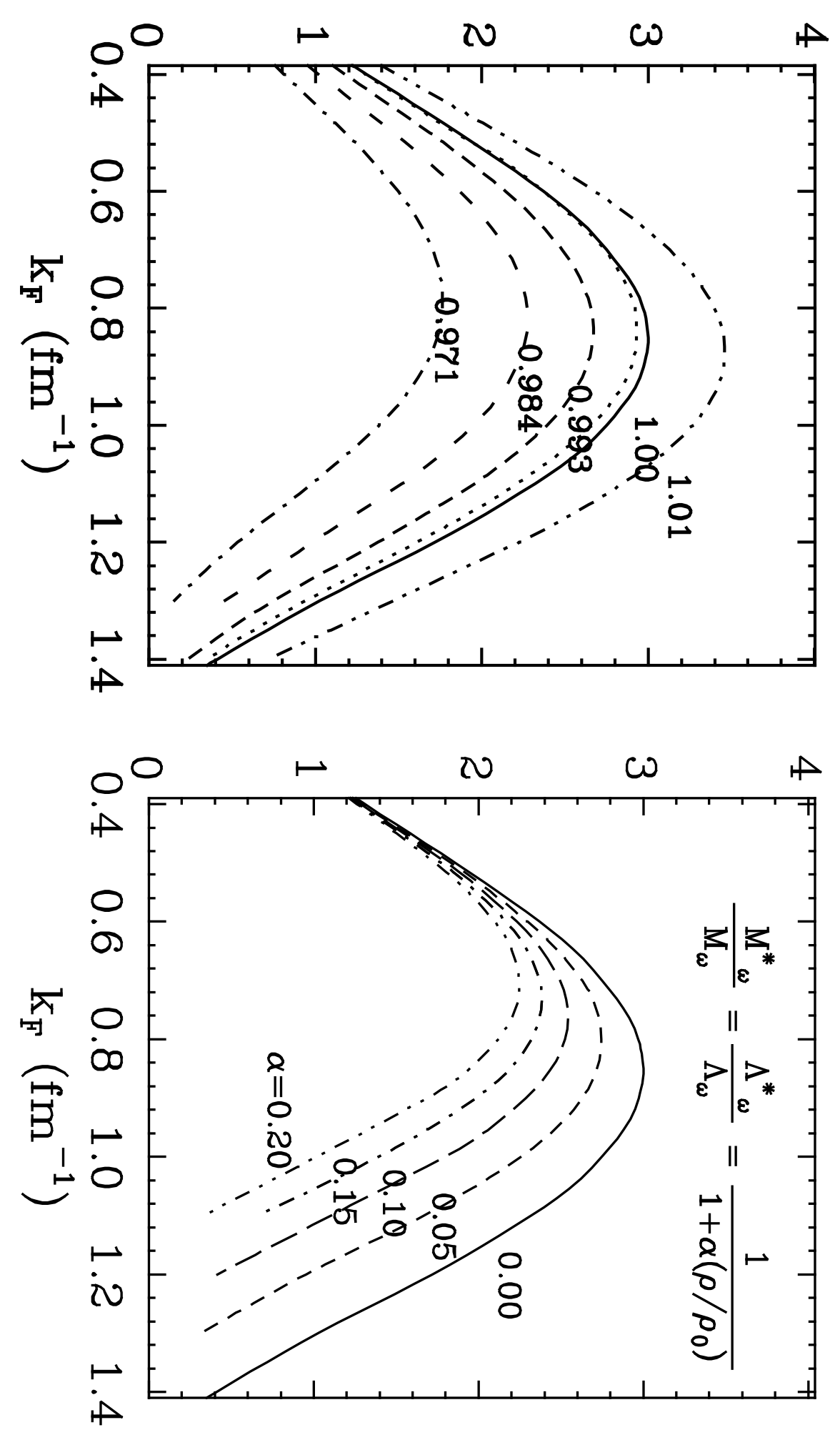